\title{
Design of RFID Production Line Visual Monitoring System
}

\author{
Liu Zhong Fu \\ College of Information \& Communication Engineering \\ Dalian Nationalities University \\ Liaoning Dalian,China \\ lzhongfu@dlnu.edu.cn
}

\author{
Wang Xia \\ Academic Service Section \\ Dalian Sport School \\ Liaoning Dalian, China) \\ 1051018029@qq.com
}

\author{
Yang YaNing \\ College of Information \& Communication Engineering \\ Dalian Nationalities University \\ Liaoning Dalian, China \\ Yyn853@dlnu.edu.cn
}

\begin{abstract}
Considering the current problems of low realtime performance for data acquisition and poor monitoring capacity in the production line monitoring system, this paper introduces a production line visual monitoring system, this system introduces the radio frequency identification technology (RFID) into the production line, and realizes the integration of RFID acquisition technology and production line control. This paper presents the system's working principles, architecture and design schemes of the software and hardware, and studies its implementation method and key technologies. The system adopts ARM9 and the embedded Linux operating system to intelligentize the operation terminal, and realizes the display interface more beautiful. The realization of multi-master methods of intelligent nodes via CAN field buses makes the system more stable. Its software and hardware design scheme, key technologies and implementation are presented also. The experimental results demonstrate that the system can be operated steadily and will have a vast application prospect.
\end{abstract}

Keywords- Monitoring System; RFID; Reader; Embedded System; Configuration Softwar

\section{INTRODUCTION}

In the manufacturing industry, if it is required to simultaneously and automatically produce different products at the same production line, it is necessary to ensure the production line identify the products at each manufacturing post and explicitly judge their status for the execution of accurate operational procedures.

The radio frequency identification (RFID) technology is a non-contact automatic identification technology realized based on the radio frequency principle. Its basic principle is that the signals realize information transmission through space coupling (alternating magnetic field and electromagnetic field) to realize the automatic identification of objects through the transmitted information[1]. Now, in the production line system of the manufacturing industry, the RFID technology provides other options, not only can it be read, it can also be written. Besides recording the product logo, it can also record the product's current status, its past and even its future status into the electronic labels[2].

An RFID system usually consists of the front-end radio frequency part and the background computer information management system. The radio frequency part consists of the reader and the electronic labels[3]. An electronic lables mark some object. Labels are implanted with IC chips and antennas, chips store the objects' information, and antennas send and receive radio waves. The reader consists of 3 parts: the radio frequency unit, the antenna and the processing unit, the main functions are: identifying information of the electronic labels through radio frequency signals; transmitting the identified information to the background hosts and then performing relevant operations accordingly[4]. Electronic labels are passive devices. It rectifies the received radio frequency pulses, supplies power after stabilizing the voltage, it provides its power supply and transmits the carrier signals to the reader.

\section{SYSTEM DESIGN}

\section{A. Working Principles}

The machining process of one product is divided into several basic procedure: each processing unit (or worker) only completes one of the procedures, and the processing unit (worker) repeats the procedure again and again machining process; the production and processing process of different products will go though one or several production lines according to the process requirements, the processed materials are transferred on the main rail of each production line, and then form the production assembly line. Meanwhile, diverge a sub rail at the processing unit where the materials or products get in and out, and the materials are automatically transferred to the correct processing unit to be processed according to the predefined processing path. Regard one workstation as a processing 
unit, bind an electronic label to each work in process before entering the production line, record the production batch and the serial number of the work in process as the unique identifier of the work in process in the whole production process. The work in process goes through each workstation in sequence according to the machining process on the production line, arrange an RFID scanning device and an intelligent terminal connected to the CAN bus at each station, the RFID scanning device identifies the label on the work in process transferred though the production line, reads the contained information and transmits it to the main control computer[5]. The main control computer updates the display information on the intelligent terminal timely according to the technological process of the current work in process, the operation terminal mainly displays the production status, process requirements, required materials, and various operation tips, and displays the commands and information transmitted from the main control computer in real time, thus assisting operating workers to be aware of production plan's status changes and execute guidance on production operations, and preventing operational errors. When the process is completed, operating workers choose to transmit the basic information of processing to the main control computer through the bus, the main control computer identifies this information and adds it to the data records correspond to the work in process. Thus through this system, the workshop management personnel's administration over the production line can drill down into each workstation, and the remote monitoring can be realized through the backbone network of enterprise information.

\section{B. System Design}

The system mainly consists of two layers of control structures, which are the upper layer of main control computer monitoring and the lower layer of control devices. The system structure of the whole monitoring system is shown in Fig .1.

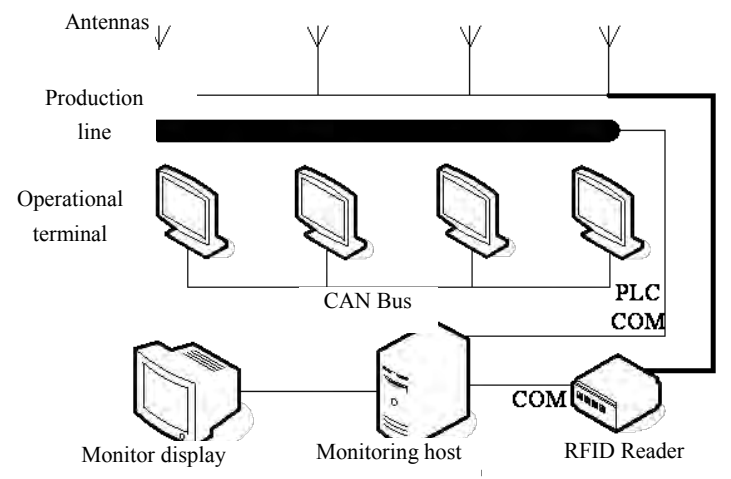

Figure 1. System Structure

\section{1) Main control computer Monitoring Layer}

The main control computer monitors the whole system through MCGS configuration software, CAN field bus devices, field devices such as the RFID reader, PLC, etc. Kunluntongtai MCGS serves as general computer system software for industrial process control and real time monitor, it has outstanding features of perfect functions, simple operations, good visibility, and strong maintainability. RFID reader reads the radio frequency label of the work in process on the production line through the antennas configured at the corresponding positions on the production line, and transmits the collected data to the MCGS configuration software monitoring platform. The main control computer adopts the ZLG_PCI9810 intelligent $\mathrm{CAN}$ interface card, this interface card adopts the standard PCI interfaces to realize the CAN2.0B protocol, establishes the connection with the MCGS configuration software through the ZOPC Server (CANBUS based general OPC server), and realizes CAN data exchange and transmission[6]. The ZOPC_Server is an OPC server application which supports to operate on all of the ZLGCAN-series interface cards; by running this server application, any client application (such as configuration software: configuration software KingView, Kun Lun on state MCGS, Intouch, etc.) supporting the OPC protocol can be run to connect to this server, ZOPC Server is the tie to realize data transmission between the $\bar{C} A N$ interface card and the configuration software[7].

When the system is running, you can choose to check the real time production status of different production lines. The main interface of the production line monitoring system MCGS is shown in Fig . 2.

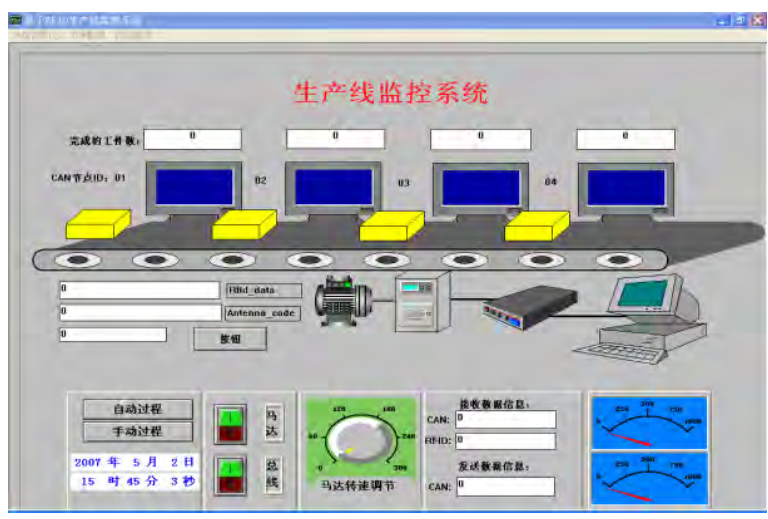

Figure 2. MCGS main interface

Display of the MCGS main interface mainly contains the main rail, workbench and main production parameters. When the workbench is working properly, it is displayed as a blue icon with link, clicking on the link can display the detailed information of the workbench, including currently processing worker's work number, login time, currently processing material, cumulative production and so on. If the workbench goes out of order, this blue icon will have red blinking outer borders, and prompt messages will be shown in the status bar, prompting the administrator to troubleshoot and remedy faults in time. When the workbench icon is displayed in grey, it indicates that the current workbench is not in use or no workbench connection exists at the current position. The administrator can send out various management instructions to workers on the production line in time through the instruction information bar at the bottom of the window. The administrator can also perform management control over the CAN bus, PLC devices, etc. through the device management window. Besides, the current time is displayed in the status bar at the bottom right corner of the main interface to help users determine the end time of order tasks conveniently[8]. 


\section{2) The Layer of Controlling Equipment}

The layer of controlling equipment mainly includes production line electrical control system, RFID reader, operational terminal of location display, etc.

The production line electrical control system adopts PLC control system and low-pressure conventional electrical control circuit; the system applies Omron $\mathrm{C} 200 \mathrm{H}$ PLC and mainly controls the start-stop and operating speed of the electrical machine, etc. RFID reader is the Sense Technology Sense-1824 UHF RFID reader, and it can independently develop the modules of the reader driving control software, build connection via OLE configuration software, and realize data exchange and transmission[9]. The reading frequency of the RFID reader is $902-928 \mathrm{MHZ}$ and its reading distance is 4.5-8.5; it has an excellent anticollision property and can reasonably meet the needs of production process in the practical operational tests. Different work stations execute some certain processing procedures based on different processing flows. Each work station will be equipped with scanning equipment with RFID and an operational terminal connected to CAN field buses; the RFID scanning equipment identifies the labels on the work pieces transferred by production lines, reads out the information on the work pieces and returns it to the main control computer for processing. In this way, the working situations in work stations can be controlled[10].

Each operational terminal is composed of CAN nodes and LCD touch screen, has functions of simple manmachine interaction, and can exhibit the real-time instructions and information passed over by the main control computer; the operator sends information to the field buses through pressing the buttons on the touch screen, and then the main control computer identifies and processes the information. The operational terminal uses Sumsang ARM mini controller as the control core, and mainly realizes the control of $\mathrm{LCD}$, the control of the touch screen, CAN communication, etc.

The S3C2410 has LCD controller in its inside, hence, it is no necessary to spread LCD controller outside. The operational terminal selects Mitsubishi 8.4-inch display screen with resolution ratio of $640 * 480$. The 4 control lines accordingly connect with the LCD control line of S3C2410, and the 18-digital data cable connects with LCD data cable of ARM. S3C241 has a built-in controller for the touch screen, and requires simple external interface electric circuits to connect with the touch screen (3). The interface electric circuits are shown in Fig .3.

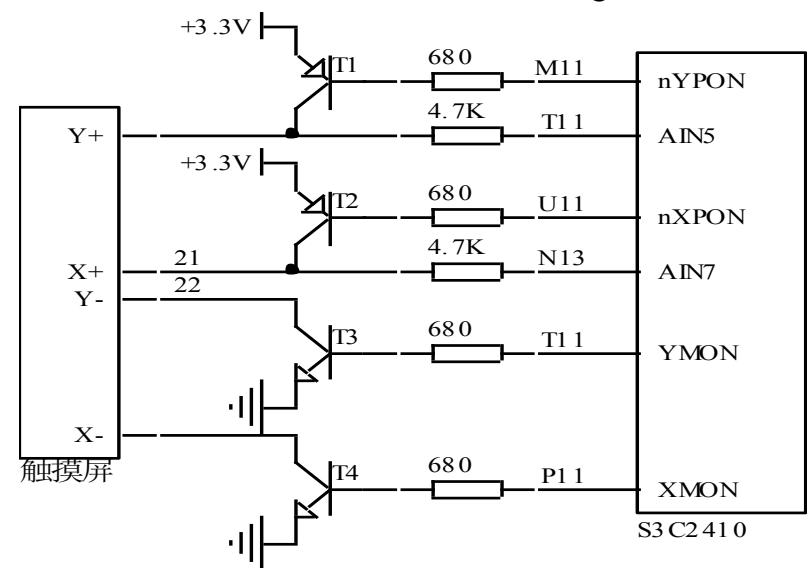

Figure 3. Touch Screen \& ARM Interface Electric Circuits
CAN modules have a data processing core of ARM mini controller- S3C2410. The inside chip of S3C2410 has no specified interface controller of CAN field buses, hence it needs to connect with CAN field bus controller via SPI interface. The CAN controller is Microchip MCP2510 and connects with S3C2410 through standards SPI interface. The Principle of interface electric circuits of CAN module is shown on Fig .4.

MCP2510 can supply power within $3-5.5 \mathrm{~V}$, and this

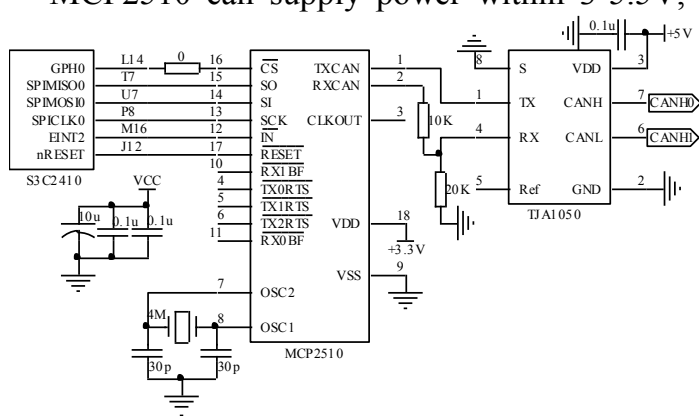

Figure 4. The Principle of interface electric circuits of CAN module

system uses $3.3 \mathrm{~V}$ voltage for power supply; hence it can directly connect with 3.3V's I/O AT91RM9200. The system employs TJA1050 as CAN bus transceiver. TJA1050 uses 5V for power supply and the problem of level compatibility arises. MCP2510 with $3.3 \mathrm{~V}$ power supply transmits the TXCAN signals with high level's minimum of $2.6 \mathrm{~V}$, and TJA 1050 with $5 \mathrm{~V}$ power supply transmits the high level with a range of $2-5.3 \mathrm{~V}$; hence, they can directly connect with each other. But when MCP2510 uses 3.3V voltage for power supply, the high level's range of RXCAN signals is $2-4.3 \mathrm{~V}$ and cannot satisfy $5 \mathrm{~V}$ 's logic level switch, hence, here we will adopt the method of divider resistance for reduction voltage, and also consider the issue of drive capability.

\section{The Design OF System SOFtware}

The whole software for the visual monitoring system includes: the design of MCGS configuration software of upper computer, that of PLC ladder diagram of the electrical control system, and that of Linux-based embedded software of the operational terminals. The operational terminals are regarding the software development. During the whole development process, the first step is to establish the cross compiling tool chain of ARM Linux, and the second step is to transplant BootLoader specific to its hardware. It is necessary to compile, reduce and transplant Linux kernel to structure Linux development environment; and the procedure above is the basis for the development of embedded software. The system design divides the software into three layers: the bottom layer is the development of Linux-based drive program for bottom-layer equipment; the middle layer is the transplantation of MiniGU; the top layer is the application program, and it's mainly responsible for the realization of users' interface display, information input and some algorithms.

Below is the introduction to communication agreements and instructions of reader and RFID labels.

RFID reader works in the on-line mode, it is a passive working mode. So, if there is a requirement for readers to 
read the real-time information on RFID labels, data communication modules must send instructions to readers every a short period of time. The instructions can be divided into rotary instructions and single instructions according to the execution times. Rotary instructions include card-reading instructions and data-sending instructions, the two types of instructions come in pairs. After receiving the card-reading instructions, readers begin trying $t$ read UID information on the card or on Block; once successful reading the information, readers will store the data in temporary memorizers; after receiving the datasending instructions, readers will transmit the data to data communication modules via CAN field buses. Single instructions are the ones that are sent out by data communication modules for some operation execution, namely: the instruction to set up the MAC addresses of readers. There are 4 types of instructions in accordance with the requirements of the whole system: the instruction to set up the MAC addresses of readers, the instruction to read UID information, the instruction to read Block Information, and the information to send data. Each instruction is composed of head marker, MAC address, operation block, content block and end marker. To identify the four different instructions, the operation blocks of the four instructions are different.

Below we will discuss the formats of each instruction.

General format: head marker (2) + MAC address (1) + data block (n) + end marker (2)

The numbers in brackets represent the number of bytes.

Firstly, we need to identify the unified head marker as FF00, end marker as 00FF, the range of MAC address as 00-FF.

The instruction format to set up MAC address is: FF 00(Original MAC) 99 (New MAC) 00 FF

For example, when we send the instruction with the content of FF 001299 D2 $00 \mathrm{FF}$, it means we reset up the reader with an original MAC address of 12 to D2. The fixed length for the instruction is 7 bytes.

The instruction format to read UID information of the tags is FF 00(MAC) DD O0 FF.

The instruction has no data block and its fixed length is 6 bytes.

The instruction to read Block information of the tags is 00(MAC) 55 (Start of block) (No. of blocks) OO FF

For example, when we send the instruction with the content of FF OO $12551 \mathrm{~F} 0 \mathrm{~A} 00 \mathrm{FF}$, it means the reader with MAC address of 12 reads the continuous 10 Block information from the 31 st block information of the TAG. The fixed length is 8 bytes. $00 \mathrm{FF}$

The instruction format to send data is FF 00(MAC) 33

The instruction has no data block and its fixed length is 6 bytes..

\section{CONCLUSION}

The essay introduces RFID technology into the production line monitoring system, realizes the seamless integration of RFID technology and production line control via the combination of field buses, upper computer monitors and lower control equipment. The experimental results demonstrate that the system can be operated steadily and will have a vast application prospect.

\section{REFERENCES}

[1] Zheng Fangwei, Zhou Mingtian, She Kun.Analyzing and comparing of mechanism for RFID information acquisition[J]. Application Research of Computers,2010,27(1):6-9,13.

[2] Xu Zhongyuan, Yuan Jiang, Qiu Zixue, et al. Distributed speed monitoring system for vehicle using RFID readers network [J] Instrument Technique and Sensor, 2010(4):33-35.

[3] Poon T C, Choy K L, Chan F T S, et al. A real-time production operations decision support system for solving stochastic production mateial demand problems [J].Expert Systems with Applications,2011,38(5):4829-4838.

[4] XU Liang, HUANG Zhiping, HE Xiaomin, CHEN Jinde. Research and Development on a Whole Process Intelligent Monitoring System of Industrial Explosive Based on FRID [J]. Explosive Materials, 2014, (2):42-47.

[5] Medeiros, Carla R. ; Costa, Jorge R. ; Fernandes, Carlos A. RFID Reader Antennas for Tag Detection in Self-Confined Volumes at UHF. IEEE Antennas \& Propagation Magazine . 2011, 53(2).

[6] LIU Long fei, ZHANG Qi-shan. Design and implementation of a long-range RFID reader[J]. The Tenth International Conference on Electronic Measurement \& Instruments. [S.1.]:ICEMI,2012:297300 .

[7] Shin, Jongmin ; Jeon, Byeongchan ; Yang, Dongmin. Multiple RFID Tags Identification with M-ary Query Tree Scheme. IEEE communications letters. 2013, 17(3).

[8] Krigslund, R. ; Dosen, S. ; Popovski, P. ; Dideriksen, J.L. ; Pedersen, G.F. ; Farina, D. A Novel Technology for Motion Capture Using Passive UHF RFID Tags. IEEE Transactions on Biomedical Engineering. 2013, 60(5).

[9] RFID in Manufacturing-A practical guide on extrac-ring measurable value from RFID mplementations in plant and warehousing operations . [2007-01-16] . http : //www.Rockwellautomation.com/solutions/rfid/get/rfidwhite.pdf.

[10] R. Rezaiesarlak ; M. Manteghi. Short-Time Matrix Pencil Method for Chipless RFID Detection Applications. IEEE Transactions on Antennas and Propagation . 2013, 61(5). 